\title{
NOTE
}

\section{Diver-operated manual suction pump sampler: a reliable method for sampling benthos on rock substrates}

\author{
Jóhann Garðar Porbjörnsson ${ }^{1,2, *}$, Jónína Herdís Ólafsdóttir ${ }^{1,2}$, \\ Bjarni Kristófer Kristjánsson ${ }^{1}$ \\ ${ }^{1}$ Hólar University College, 551 Sauðárkrókur, Iceland \\ ${ }^{2}$ Marine and Freshwater Research Institute, 101 Reykjavík, Iceland
}

\begin{abstract}
Although numerous methods have been developed for SCUBA diver-operated sampling, few are suitable for sampling complex physical substrates. Sites such as groundwater springs sometimes have narrow sections along with uneven vertical and horizontal rock substrates at various depths. Here we developed a SCUBA diver-operated suction pump sampling system, designed for the sampling of benthic material on morphologically complex substrates. The sampler was designed to be simple to use in an already gear-heavy operation. The device is attached to the diver, making it a suitable tool for work on vertical substrates, and can be operated by 1 person. We compared the macrozoobenthic sampling yield and usability of the suction pump sampler to a Surber sampler that was modified for use while diving. Samples taken with the suction pump sampler had an overall greater macrozoobenthic density and species richness than samples taken with the modified Surber sampler. Sampling was more efficient with the suction pump sampler, as sampling containers were easily replaced underwater, whereas the modified Surber needed to be transported to the surface and its sample fixed before the next sample could be taken. Various modifications of the suction pump sampler are possible, depending on the objective of the sampling process, and it can be constructed with inexpensive and readily available materials. The suction pump sampler presented here further allows researchers in the field of marine and freshwater ecology to accurately sample the benthic habitat, including habitats where physical complexity may previously have prevented sampling.
\end{abstract}

KEY WORDS: Benthic habitats - SCUBA diving - Benthic diving - Methods - Sampling · Rock substrate $\cdot$ Hard-substrate assemblages $\cdot$ Benthos $\cdot$ Epifauna

\section{INTRODUCTION}

A key to systematically recording and monitoring aquatic ecosystems is the access to accurate sampling methods (Dodds 2002, Hauer \& Lamberti 2017). In the benthic habitat, soft bottom is commonly sampled with grab or core samplers, intended for the removal of substrate (Radtke 2005, Hauer \& Lamberti 2017), and freshwater stream benthos are commonly sampled with Surber or kick-net samplers (Kroger

*Corresponding author: johanng@hafogvatn.is
1972, Hauer \& Lamberti 2017). These methods have been popular, although their operation is traditionally restricted to shallow depths where currents are present (Barbour et al. 1999). Another common method for sampling hard bottom is to remove stones and scrub them with a soft brush in a water-filled bucket, after which the dislodged material is collected in a sieve (Hauer \& Lamberti 2017). However, such loose rocks are not always available. Sampling hard continuous substrates is problematic (Barbour

(C) The authors 2018. Open Access under Creative Commons by Attribution Licence. Use, distribution and reproduction are unrestricted. Authors and original publication must be credited. 
et al. 1999) and poses a greater challenge when the substrate is deep and/or vertical. For deeper sites, sampling is often carried out by SCUBA divers, most commonly using quadrats, hand collecting and photo surveying (OSPAR Commission 2012), but numerous other methods have been specifically developed for the sampling of hard substrates accessed by SCUBA divers. Runnels (1985) presented a method for the quantitative collection of invertebrates in benthic areas, where animals were brushed off the bottom in a sealed box and subsequently vacuumed through a hose and into a sealed sampling jar. However, this method depends on an air supply, either from the diver or a separate air tank, which reduces portability and complicates sampling. In addition, it requires substrates to be flat enough so that the device can properly seal off the sampling area. Later, Lønne (1988) developed an electronic suction pump for sampling sympagic fauna living under ice. This method requires a battery, which limits its underwater sampling time to $20 \mathrm{~min}$ at near freezing temperatures. To be able to carry out continuous sampling in a portable manner, manual systems are better suited. In that regard, Andrulewicz et al. (2004) presented a modified Krautsky sampler wherein a metal frame is attached to a collection bag with a sleeve. This way divers can remove material that gathers inside a collection bag. However, repetitive sampling would require multiple samplers or direct transport of the sampler to the surface for the collection bag to be emptied. Gulliksen \& Derås (1975) described a system for sampling sessile macrozoobenthos. This system allows for the collection of several samples during each dive and can be used on vertical substrates. However, for samples that consist of fine organic matter, there is a risk of losing part of the sample, especially between the time when sampling is discontinued and the container is replaced.

The method presented here builds upon previously developed methods to facilitate the sampling of macrozoobenthos and biofilm material in groundwaterfilled rift fissures in Iceland in 2013 (Ólafsdóttir 2015). The fissures have cold $\left(3-4^{\circ} \mathrm{C}\right)$ groundwater (Ólafsson 1992) and both vertical and horizontal substrates. Our project involved sampling carried out with SCUBA diving, as the sampling stations were located at a depth ranging from $1-30 \mathrm{~m}$. Since some of the fissures have difficult land access, we needed a portable sampling device that would allow us to take several samples during each dive. Further, we needed to be able to collect quantitative samples on both horizontal and vertical rock substrates. As our objective was to sample macrozoobenthos and the fine benthic biofilm, we needed to ensure that we had an accurate sampling method that would prevent any of the sample from spilling into the surrounding water column. We also needed the sampling device to be as simple as possible to use in an already gear-heavy operation. Furthermore, we needed a portable system made of inexpensive, readily available materials, that could be attached to a diver enabling effortless maneuvering in narrow spaces and/or deep water where there is considerable distance to the surface. With the above considerations in mind, we designed a manual diver-operated suction pump sampling system intended for sampling on complex substrates, such as vertical and horizontal walls along narrow sections in groundwater fissures. We compared the usability and sampling yield of the suction pump sampling system with a Surber sampler modified to be used while diving.

\section{MATERIALS AND METHODS}

We tested the usability and sampling yield of the suction pump sampler and the modified Surber sampler in the groundwater fissure Silfra in Pingvellir National Park, southwest Iceland, in July 2013. Samples were taken at 2 sites at different depths (1 and $10 \mathrm{~m})$. Vertical fissure walls were sampled at $1 \mathrm{~m}$, whereas the deeper site $(10 \mathrm{~m})$ had a horizontal bottom. At each sampling site, 5 replicate samples were taken with each method. On each 45 min dive, we were able to collect 10 samples with the suction pump sampling system while 5 samples were taken with the modified Surber.

The suction pump sampling system was designed and built using a single acting bilge pump (Munster Simms Engineering), which was attached with hose clamps to 2 flexible $3 \mathrm{~cm}$ diameter plastic hoses (45 and $190 \mathrm{~cm}$ long) on either end (Fig. 1). The longer hose was used for the collection of sampled material, while the shorter hose could be screwed to a valve that was fastened to the lid of a $1.9 \mathrm{l}$ sampling container. The sampling container was made of 2 PVC pipes that were fitted together $(10.5 \mathrm{~cm}$ diameter, $22 \mathrm{~cm}$ length) with a $125 \mu \mathrm{m}$ mesh at the downstream end of the container (Fig. 2). The upstream end of the container was fitted with a lid and valve for the prevention of any material from flowing back out of the system. While closed, the sampling container was easily unscrewed and replaced underwater, so that several samples could be collected on a single dive. Sampling containers were collected into a net bag and stored there until the end of each dive. For sam- 


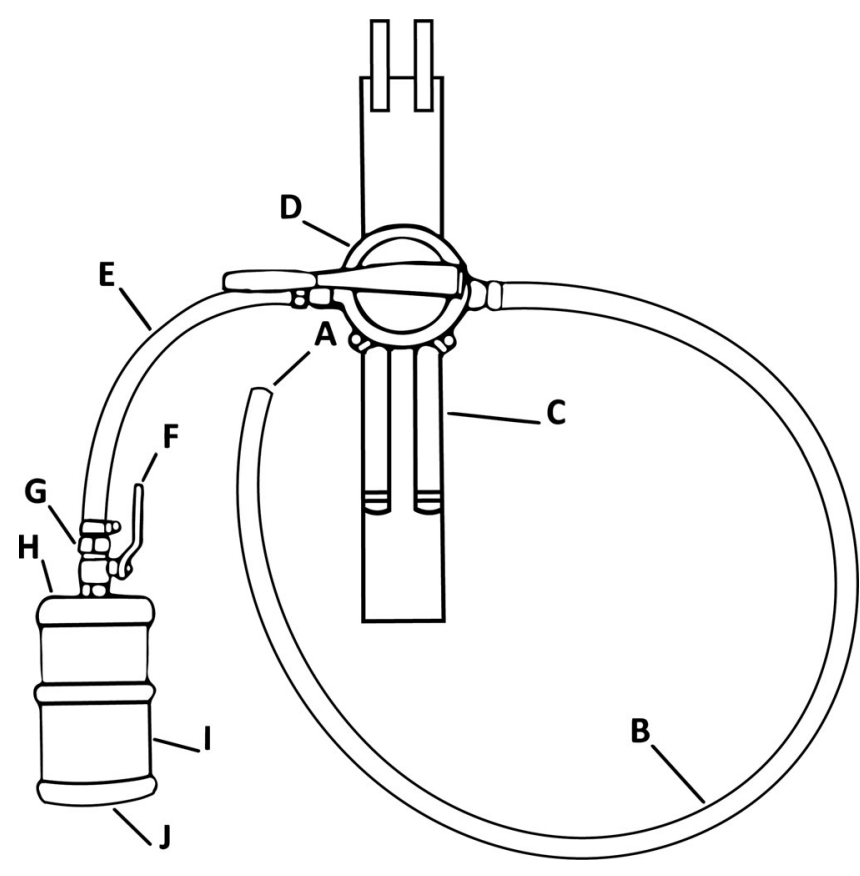

Fig. 1. Diver-operated manual suction pump sampler designed for benthic sampling in aquatic systems. (A) Opening of a long $(190 \mathrm{~cm})$ flexible plastic hose (B) where a sample enters through suction. (B) The hose is fastened to a bilge pump (D) with hose clamps, for the suction of material from a sampling site into the sampling container (I). (C) A sewn harness with buckles to thread around the waist of the operator. (D) Manual bilge pump (Munster Simms Engineering). (E) Short $(45 \mathrm{~cm})$ flexible plastic hose that can be screwed onto the sampling container (I). (F) Manual valve system operated with a handle for secure containment of a sample within the sampling container (I). (G) Screw-gate nut for attaching the short hose (E) to the valve of the sampling container $(\mathrm{F})$. $(\mathrm{H})$ Lid for closing the sampling container. (I) Sampling container (1.9 l volume). (J) Mesh net (125 $\mu \mathrm{m})$ fastened to the sampling container by fitting 2 PVC pipes together

pling, a diver attaches the pump around the waist with a harness and then pumps continuously to collect material at a desired site (Fig. 3). For our purposes, a $20 \times 20 \mathrm{~cm}$ stainless steel quadrat was used for quantitative sampling. One diver operated the pump and held the quadrat at the desired sampling site. The other diver dislodged benthic material with a soft brush and collected it with the long hose of the sampler. Once the material had been completely brushed from inside the quadrat, the valve connecting the plastic hose to the container was closed and the container unscrewed from the system. The closed container was then placed in the net bag. The system was rinsed by placing a fine mesh, carried by the diver, at the opening of the longer hose and pumping sieved water through the system. If further sampling

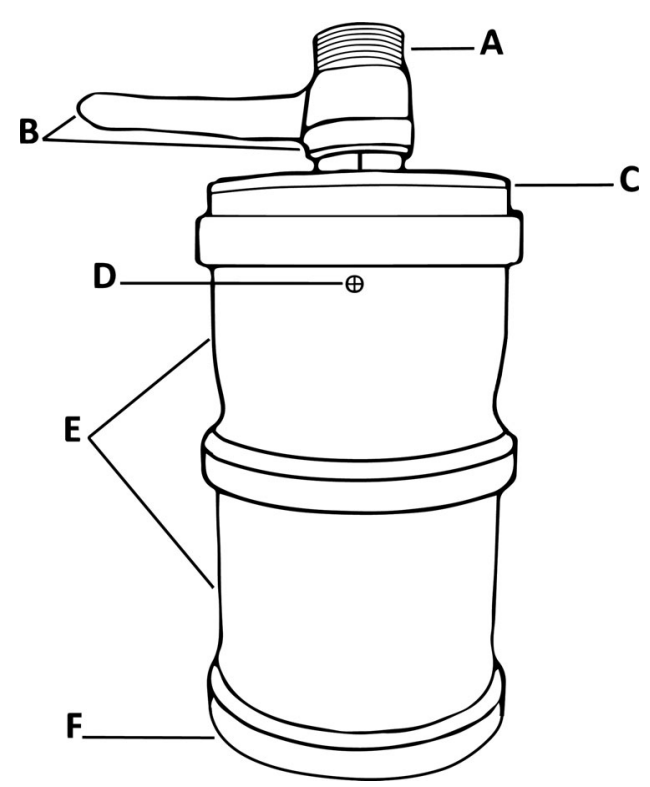

Fig. 2. Plastic sampling container for a diver-operated manual suction pump sampler. (A) Threads for a screw-gate nut on a short flexible plastic hose of the manual diver-operated suction pump sampler ( $\mathrm{G}$ and $\mathrm{E}$ in Fig. 1). (B) Manual valve operated with a handle for a secure containment of a sample within the sampling container. (C) Lid for the sampling container. (D) Screw for securing the lid (C). (E) Two $10 \mathrm{~cm}$ long PVC pipes snugly fitted together forming the $1.9 \mathrm{l}$ container.

(F) Mesh $(125 \mu \mathrm{m})$ on the bottom of the container

was desired, an empty container was screwed on to the suction pump system for the next sample. Once all samples had been collected, they were brought to the surface and removed from the sampling container by washing them into a 0.51 plastic canister using filtered water from a squeeze bottle.

For comparison, a Surber sampler (EFE\&GB Nets), commonly used in freshwater systems, with a collection area of $20 \times 20 \mathrm{~cm}$ was modified to be used while diving (Fig. 4). The modification ensured that the Surber's quadrat could be closed underwater to prevent sample spilling. Two divers participated in the sampling; one diver brushed and guided material into the Surber's mesh net, while the other made sure the Surber stayed open and further held the quadrat in place. The Surber was placed randomly on hard substrate at each depth. With its opening facing upstream, the sampling area inside the Surber's quadrat was brushed with a soft brush to release material which was directed by the current into the Surber's mesh net $(65 \mu \mathrm{m})$. The Surber was then closed with watertight fabric of a mesh size of $<65 \mu \mathrm{m}$ that was locked in place by folding the Surber's frame together. The modified Surber and its sample was subsequently brought up to the surface. 

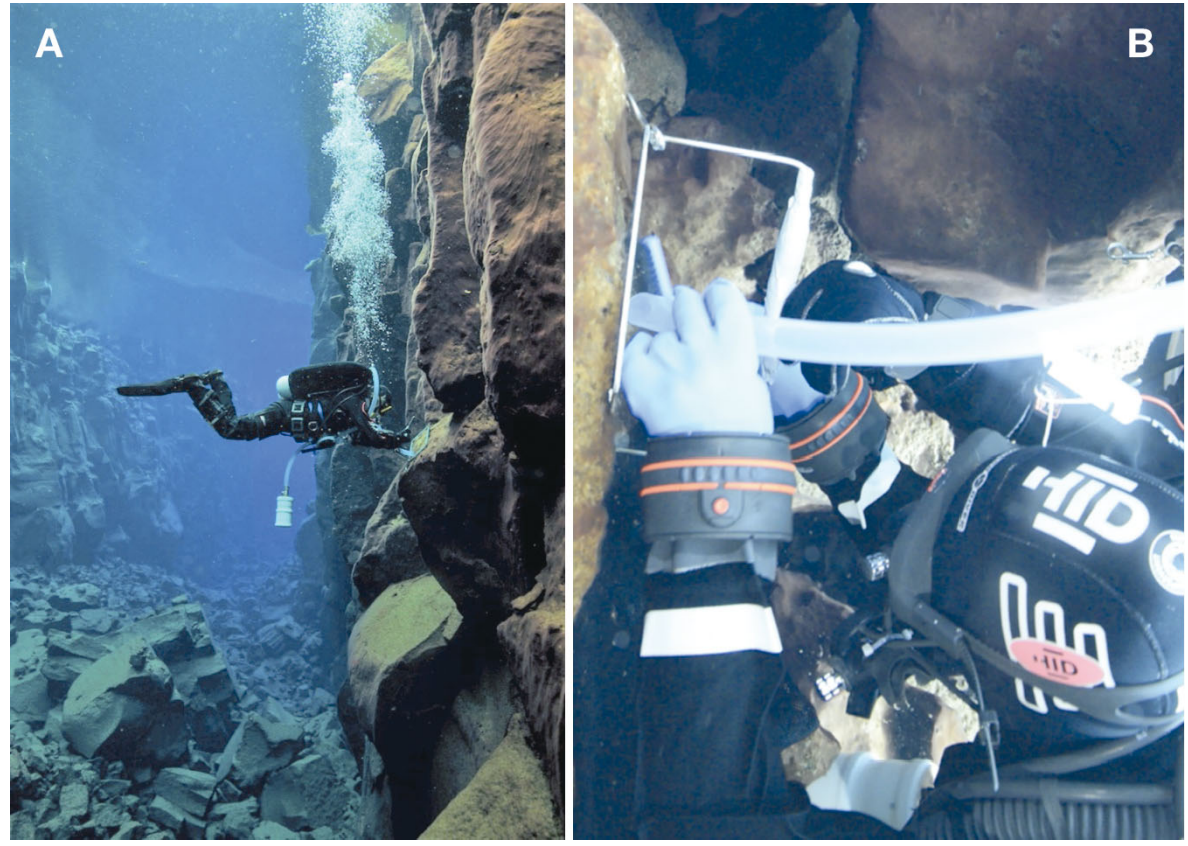

Fig. 3. (A) Diver using the diver-operated manual suction pump sampler on a vertical rock wall. (B) Two divers working together to sample benthic material from a vertical rock wall. One diver has the suction pump sampler harnessed to his/her body and holds a frame in place while the other diver gently brushes the sampling site and collects dislodged material with the longer hose of the sampler. Photos by Gísli Arnar Guðmundsson

At the $1 \mathrm{~m}$ depth station, the diver surfaced and handed the Surber containing the sample to the surface assistant, who then sifted the sample through a $125 \mu \mathrm{m}$ mesh sieve to make sure only organisms within the desired size range would be collected. At the $10 \mathrm{~m}$ depth station, a rope was attached to the Surber on which the diver pulled 3 times, signaling the surface assistant to pull up the Surber and its

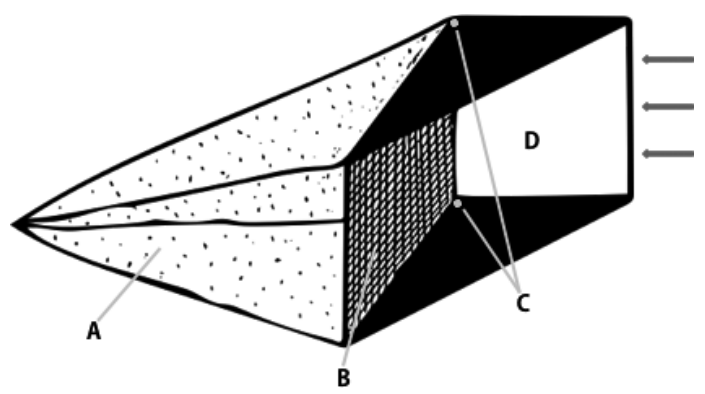

Fig. 4. Modified Surber sampler for use while SCUBA diving. (A) Mesh net $(65 \mu \mathrm{m})$. (B) Watertight fabric $(<65 \mu \mathrm{m})$ that was taken aside while sampling and then placed over the Surber's opening once sampling was finished. (C) Hinges that allowed the sampling frame to be folded backwards towards the opening of the Surber. By folding the frame backwards, the watertight fabric was secured in place which allowed the transport of the Surber up to the surface. (D) Sampling frame $(20 \times 20 \mathrm{~cm})$. Arrows indicate the direction of the current sample. This was done so divers did not need to do multiple ascents which may increase the chance of developing decompression sickness (Gorman 1989). The 2 divers remained at $10 \mathrm{~m}$ depth until the sample had been processed and the Surber lowered back to the divers.

Sampled organisms collected with both methods were fixed in $70 \%$ ethanol, brought to a laboratory and identified and counted under a dissecting microscope. In order to compare the sampling yield of the 2 methods, a Mann-Whitney $U$-test was used. Numerical analysis was done in Microsoft Excel 2011 for Mac and SPSS (IBM, version 14.0). Graphics were drawn in Adobe Illustrator CC 2018 for Windows (Adobe Systems).

\section{RESULTS AND DISCUSSION}

Our objective was to construct a portable sampler made of inexpensive, readily available materials suitable for accurate sampling of sessile benthic material on morphologically complex substrates, such as vertical and horizontal rock walls. Samples collected with both the suction pump sampler and the modified Surber contained numerous taxa of macrozoobenthos (Table 1) and biofilm material. Samples taken 
Table 1. Mean (SD) taxon density in samples collected with 2 samplers, a modified Surber and a diver-operated manual suction pump sampler, within a $20 \times 20 \mathrm{~cm}$ area at 1 and $10 \mathrm{~m}$ depth in Silfra, Thingvellir National Park, Iceland

\begin{tabular}{|lrccc|}
\hline & \multicolumn{3}{c}{ Modified Surber sampler } & \multicolumn{2}{c|}{ Suction pump sampler } \\
& $1 \mathrm{~m}$ & $10 \mathrm{~m}$ & $1 \mathrm{~m}$ & $10 \mathrm{~m}$ \\
\hline Acarina & $0.2(0.40)$ & $0(0.00)$ & $0.2(0.40)$ & $1.6(1.62)$ \\
Copepoda & $0.2(0.40)$ & $1.4(1.74)$ & $0(0.00)$ & $6.8(3.25)$ \\
Terrestrial & $0.8(0.75)$ & $0.0(0.00)$ & $0(0.00)$ & $0.0(0.00)$ \\
Chironomidae pupae & $1.6(1.20)$ & $0.0(0.00)$ & $2.0(1.10)$ & $2.4(1.85)$ \\
Chironomidae larvae & $36.8(7.30)$ & $7.6(4.63)$ & $52.0(23.13)$ & $42.8(15.80)$ \\
Ostracoda & $0.2(0.40)$ & $0.0(0.00)$ & $8.4(0.00)$ & $0.0(0.00)$ \\
Plecoptera & $0.2(0.40)$ & $0.0(0.00)$ & $0.0(0.00)$ & $0.0(0.00)$ \\
Cladocera & $0(0.00)$ & $0.2(0.40)$ & $0.8(1.17)$ & $1.8(1.94)$ \\
& & & & \\
\hline
\end{tabular}

be adjusted, cameras need to be operated for photo surveying, etc. During our testing, having the sampler harnessed to the diver did not affect the operation of dive equipment in a backmount setup. By installing a valve to the sampling container, sample spilling was prevented which ensured easy replacement of sampling containers underwater, facilitating repetitive sampling. Depending on the objective, 1 or 2 divers can operate the system; a single diver can conduct sampling on loosely attached organisms and sediments that can be removed from the substrate by suc-

with the suction pump sampler contained higher species richness $(U=17.5, \mathrm{p}<0.05)$ and density $(U=$ 9.5, $\mathrm{p}<0.01)$ compared to samples taken with the modified Surber sampler. When comparing each depth independently, the differences were insignificant for density $\left(U_{1 \mathrm{~m}}=4, \mathrm{p}>0.05 ; U_{10 \mathrm{~m}}=0, \mathrm{p}>0.05\right)$, and significant for species richness at $10 \mathrm{~m}(U=1, \mathrm{p}<$ $0.05)$ but not at $1 \mathrm{~m}(U=8, \mathrm{p}>0.05)$. The differences are likely a result of inaccurate sampling while using the modified Surber sampler, as it was observed that material that had been brushed off the substrate was inadequately collected in the mesh bag, likely because of insufficient current. In contrast, the suction pump sampler was well suited for sampling on vertical rock wall and horizontal bottom, and biofilm material was easily removed from the substrate, allowing us to collect samples of micro-, meio- and macrobenthos. In addition, the sampling process was more efficient while using the suction pump sampler: 5 quadrats were sampled during a 45 min dive while using the modified Surber compared to 10 quadrats while using the suction pump sampler. This is reflected in the fact that transporting the Surber up and down from each depth station and waiting for the sample to be processed was a timely process.

The suction pump sampler presented here builds upon previously developed methods used by SCUBA divers for sampling. Here, 2 novel features have been included in the design of the sampler: a harness to attach the sampler directly to the diver and a valve on the sample container to prevent sample spilling. Depending on sampling protocols and needs, having the sampler harnessed to the diver can provide greater portability and freedom to operate diving equipment in mid-water. This is particularly useful when sampling vertical walls where there is nowhere to place the sampler when dive gear needs to tion alone, while 2 divers are required when a brush is needed to remove organisms from the substrate. The sampler can be easily modified in the field, depending on target organisms, the sampling environment and protocols. We installed a $125 \mu \mathrm{m}$ sieve in the sampling container, but the sampler can be equipped with different mesh sizes by simply separating the 2-piece container and inserting a new mesh. Similarly, the container can be made smaller or larger with readily available PVC pipes. Many other modifications are possible. The length of the flexible plastic hoses can be modified depending on the need for reach, and the sampling frame can also be modified, e.g. by size or by using a frame from flexible material that better seals and encloses the sampling area.

Generally, the sampler was easily operated, but like in any other system using a sieve, the mesh occasionally got clogged. To avoid this, wider PVC pipes could be used for the construction of the sampling container, thus increasing the mesh area. Occasionally, sampled organisms got stuck on the boundary of the mesh and the lower PVC pipe. This may be hindered by sealing the mesh to the container, such as by applying silicon on both the inside and outside mesh. If used in future studies, the sampler should be equipped with a double acting pump instead of a single acting pump like the one presented here. This would further prevent organisms from being released while pumping takes place.

The diver-operated manual suction pump sampler described here can be reliably used on morphologically complex substrates when sampling benthic materials in aquatic environments. As such, it enables researchers to accurately sample the benthic habitat, even habitats where substrate complexity may previously have prevented sampling. 
Acknowledgements. Thanks to Arnpór Gústavsson for his help with constructing and designing the suction pump sampler, Kjartan Guðmundsson and Valgeir Pétursson for their assistance during sampling and Jón S. Ólafsson for constructive criticism. Finally, we thank the National Geographic Society and the Rannís Icelandic Student Innovation Fund for supporting the project.

\section{LITERATURE CITED}

Andrulewicz E, Kruk-Dowgiallo L, Osowiecki A (2004) Phytobenthos and macrozoobenthos of the Slupsk Bank stony reefs, Baltic Sea. Hydrobiologia 514:163-170

Barbour MT, Gerritsen J, Snyder BD, Stribling JB (1999) Rapid bioassessment protocols for use in streams and wadeable rivers: periphyton, benthic macroinvertebrates and fish, $2^{\text {nd }}$ edn. Office of Water, US Environmental Protection Agency, Washington, DC

Dodds WK (2002) Freshwater ecology: concepts and environmental applications. Academic Press, San Diego, CA

Gorman DF (1989) Decompression sickness and arterial gas embolism in sports scuba divers. Sports Med 8:32-42

* Gulliksen B, Derås KM (1975) A diver-operated suction sam-

Editorial responsibility: Anna Metaxas,

Halifax, Nova Scotia, Canada pler for fauna on rocky bottoms. Oikos 26:246-249

Hauer FR, Lamberti GA (2017) Methods in stream ecology, $3^{\text {rd }}$ edn, Vol 2. Academic Press, San Diego, CA

Kroger RL (1972) Underestimation of standing crop by the Surber sampler. Limnol Oceanogr 17:475-478

Lønne OJ (1988) A diver-operated suction sampler for sympagic (= under-ice) invertebrates. Polar Res 6:135-136

Ólafsdóttir JH (2015) Biological diversity in groundwater fissures within the Icelandic neovolcanic zone. MSc thesis, Hólar University College, Sauðárkrókur

Ólafsson J (1992) Chemical characteristics and trace elements of Thingvallavatn. Oikos 64:151-161

OSPAR Commission (2012) Hard-bottom macrophytobenthos, soft-bottom macrophytobenthos and hard-bottom macrozoobenthos. In: JAMP eutrophication monitoring guidelines: benthos. OSPAR Commission, Bonn, p 8-11

Radtke DB (2005) Bottom-material samples. In: US Geological Survey Techniques of Water-Resources Investigations, Book 9. US Geological Survey, Reston, VA, p 18-19

Runnels RJ (1985) Diver sampling of macroinvertebrates on northwestern Gulf of Mexico hard bottom areas. In: Mitchell CT (ed) Diving for science. Proc AAUS/ CMAS Annual Scientific Diving Symp. La Jolla, CA, p 115-122

Submitted: December 13, 2017; Accepted: July 10, 2018

Proofs received from author(s): August 31, 2018 